01

\title{
Квантовая модель диполь-индуцированный диполь и влияние инертного окружения на интенсивность ИК поглощения
}

\author{
() А.П. Коузов ${ }^{1}$, Н.И. Егорова ${ }^{2}$, А.Н. Добротворская ${ }^{3}$ \\ ${ }^{1}$ Санкт-Петербургский государственный университет, \\ 198504 Санкт-Петербург, Россия \\ ${ }^{2}$ Санкт-Петербургский университет ГПС МЧС России, \\ 196105 Санкт-Петербург, Россия \\ ${ }^{3}$ ЗАО НПО „Ленкор“, \\ 196084 Санкт-Петербург, Россия \\ e-mail: alex@ak1197.spb.edu
}

Поступила в редакцию 25.02.2019 г.

В окончательной редакции 22.04.2019 г.

Принята к публикации 23.04.2019 г.

На основе диаграммной техники развита теория мнимой части изотропной поляризуемости, индуцированной диполь-дипольными взаимодействиями между изотропно поляризующимися частицами. Предложена классификация однократных ИК резонансов, отвечающих случаям разрешенного поглощения и поглощения, индуцированного межмолекулярными взаимодействиями. Для схемы диполь-индуцированный диполь (DID) проведено суммирование диаграмных вкладов, описывающих изменение интенсивности разрешенного поглощения из-за эффекта реактивного поля окружения. Полученные таким образом строгие результаты противоречат полуэмпирической модели Онзагера-Беттчера. Проведенные численные оценки показывают, что роль реактивного поля в наблюдаемых изменениях интенсивностей колебательных полос простейших модельных систем ( $\mathrm{HCl}$ и $\mathrm{HBr}$ в инертных растворителях) мала и уменьшается с ростом размера частиц растворителя, что указывает на неадекватность моделей DID и Онзагера-Беттчера данным опыта.

Ключевые слова: молекулярная электрооптика, межмолекулярные взаимодействия.

DOI: $10.21883 /$ OS.2019.08.48031.70-19

\section{Введение}

Изменение интенсивности молекулярных ИК полос поглощения при увеличении плотности буферных (т.е. спектроскопически неактивных) частиц является интересным эффектом, до сих пор плохо поддающимся количественной интерпретации. Поскольку этот эффект есть результат фундаментальных процессов, обусловленных динамикой межмолекулярных взаимодействий (MМB) в плотных средах, его теоретическая картина важна c академической точки зрения и может также найти применение в физике планетных атмосфер со значительной плотностью газа (Венера) или с заметным содержанием частиц жидких или твердых фракций (Земля). Теоретический анализ естественно начать с простейших модельных систем, каковыми являются криорастворы галоидоводородов, для которых собран достаточно надежный и многообразный экспериментальный материал по интенсивностям колебательных полос поглощения [1].

Для интерпретации изменения интегрального коэффициента поглощения $A$ буферным растворителем могут быть привлечены два хорошо известных и условно независимых эффекта. Первый связан с отличием падающего поля $\mathbf{E}_{\mathbf{0}}$ от внутреннего (микро)поля $\mathbf{E}$, непосредственно взаимодействующего с дипольным моментом молекулы. Учет такого отличия приводит к масштабированию $A$ стандартным фактором Пола-Уилсона, что далеко недо- статочно [2] для объяснения изменения $A$ в упомянутых криорастворах.

Кроме того, из-за наличия ММВ эффективный дипольный момент $\mathbf{D}$, фигурирующий в операторе взаимодействия молекула-поле $(-(\mathbf{D}, \mathbf{E}))$, может отличаться от собственного диполя d примесной молекулы, что связывается с существованием так называемого реактивного поля (RF): поле диполя $\mathbf{d}$ поляризует окружение, и эта поляризация служит источником $\mathrm{RF} \mathbf{E}^{(R F)}$, наводящего на примесной молекуле дополнительный дипольный момент $\Delta \mathbf{d}$, так что $\mathbf{D}=\mathbf{d}+\Delta \mathbf{d}$. При учете $\mathrm{RF}$ величина $A_{f i}$ полосы колебательного перехода $f \leftarrow i$ будет определяться усредненным по термостату квадратом матричного элемента вектора D:

$$
\begin{aligned}
A_{f i} \propto & \overline{\left|\mathbf{D}_{f i}\right|^{2}}=\left(\mathbf{d}_{i f}, \mathbf{d}_{f i}\right)+\left(\mathbf{d}_{i f}, \overline{\Delta \mathbf{d}_{f i}}\right) \\
& +\left(\overline{\Delta \mathbf{d}_{i f}}, \mathbf{d}_{f i}\right)+\left(\overline{\Delta \mathbf{d}_{i f}, \Delta \mathbf{d}_{f i}}\right)
\end{aligned}
$$

Отметим также, что обратная поляризация может проявляться и в ван-дер-ваальсовых комплексах, влияя на интенсивность разрешенных переходов в партнерах.

Эмпирические характеристики $\mathbf{E}^{(R F)}$ и $\overline{\Delta \mathbf{d}}$, равно как и радиус ячейки Онзагера $r$, используемые в теории диэлектрической постоянной [3,4] и поглощения [2], имеют смысл статистически средних по окружению. Сразу отметим, что включение в расчет [2] квадратичного слагаемого $\left(\overline{\Delta \mathbf{d}_{f i}}\right)^{2}$ и слагаемых, нелинейных 
по RF, противоречит флуктуационно-диссипационной теории [5], которая выражает коэффициент поглощения через усредненный квадрат дипольного момента, а не через квадрат его среднего. По этой причине расчеты [2], использовавшие ячеечную модель RF ОнзагераБеттчера [4] с варьируемым радиусом ячейки $r$, нуждаются в коррекции. Примечательно, что оптимальные значения $r$ [2] оказались примерно в два раза меньше радиуса первой координационной сферы, что прямо указывает на неадекватность опыту самой ячеечной модели $\mathrm{RF}$. При реалистических значениях $r$ роль слагаемых, нелинейных по $\overline{\Delta \mathbf{d}_{f i}}$, становится пренебрежимо малой, что исправляет некорректность моделирования, но в этом случае остающиеся (линейные) поправки к интенсивности сильно недооценивают экспериментальные значения.

Следуя классической схеме диполь-индуцированный диполь (DID) [6] и постулируя существование двух RF в пространстве между двумя взаимодействующими атомами, Фроммхольд [7] рассчитал индуцированную часть нерезонансной (электронной) поляризуемости $\Delta \alpha(R)$. Из его формул также можно сделать вывод о слабости RF-эффекта при статистически значимых межатомных расстояниях $R$.

Таким образом, разработанные к настоящему времени теоретические модели изменения интенсивности основаны на представлениях классической электрооптики и неспособны дать количественную интерпретацию опытных данных. Кроме того, недостатком теории Онзагеpa $[3,4]$ является применение континуальной модели ко всему объему диэлектрика, в то время как отклик от слоев, прилегающих к активной молекуле, должен учитывать их микроструктуру.

Целью данной работы является квантово-механический анализ проблемы, основанный на теории возмущений (ТВ). Из общих соображений можно ожидать, что по мере перехода в более высокие порядки ТВ квантовые результаты будут все сильнее отклоняться от классической картины поляризации. Для их визуализации крайне полезным является диаграммное рассмотрение [8-11] мнимой части поляризуемости $\Delta \alpha$, индуцированной мгновенными диполь-дипольными (DD) взаимодействиями между линейной молекулой и ее окружением из сферически симметричных частиц. Такой подход приводит к естественной классификации спектральных вкладов на разрешенные и индуцированные (см. ниже). Более того, он помогает выявить существование не только однократных, но и многократных резонансов в индуцированной восприимчивости. Рассмотрение последних, однако, требует учета влияние термостата на колебательно-вращательные степени свободы и в рамках данного подхода не может быть проведено до конца.

Анализ однократных резонансов значительно упрощается, если пренебречь вращательной структурой полосы и оперировать с единственной (эффективной) частотой $\omega_{f i}$ и соответствующей (феноменологической) константой затухания $\gamma_{f i}$, что и будет использоваться при дальнейших расчетах. Переход к более реалистичному описанию потребовал бы не только учета структуры полосы, но и введения точной вращательной релаксационной матрицы на основе более сложной техники Лиувилля [12-15], что планируется сделать в будущем.

Отметим также, что поглощение света в оптически однородной среде [16] определяется мнимой часть изотропной поляризуемости ансамбля взаимодействующих молекул. По этой причине DD-механизмы индукции анизотропной поляризуемости в нашей работе не рассматриваются.

\section{Классификация диаграммных вкладов в интенсивность поглощения}

На диаграммах, изображающих различные вклады в бинарную индуцированную поляризуемость, пара вертикальных линий дает эволюцию состояний частиц, возмущаемую DD-взаимодействиями в ближней зоне (горизонтальные сегменты) и процессами поглощения (входящая стрелка) и испускания (выходящая стрелка) фотонов той или иной частицей. Правила составления выражения для амплитуды рассеяния, даваемой конкретной диаграммой, стандартны и описаны в работе [9]. Необходимо также учитывать, что, согласно ТВ, виртуальные состояния должны отличаться от начального состояния системы $|I\rangle=\left|i_{a}\right\rangle\left|i_{b}\right\rangle\left|i_{t r}\right\rangle|N\rangle$, определяемого векторами исходных состояний партнеров $a$ и $b$, их относительного трансляционного движения $(t r)$ и поля с числом фотонов $N$. По завершении всех взаимодействий система восстанавливает начальное состояние.

Вклады можно разделить на собственные, когда поглощение и излучение осуществляются одной и той же частицей, и перекрестные, когда связь с фотонами затрагивает обе молекулы. Изотропия пространства накладывает определенные ограничения. Так, если число $n$ DDсегментов нечетно, то существуют лишь перекрестные вклады, а собственные вклады, отличные от нуля, даются диаграммами с четными $n$.

Используемое далее адиабатическое приближение предполагает следующую иерархию частот электронных $(e l)$, колебательных $(v)$, вращательных $(r)$ и трансляционных (tr) переходов: $\left|\omega_{\mathrm{el}}\right| \gg\left|\omega_{v}\right| \gg\left(\left|\omega_{r}\right|,\left|\omega_{t r}\right|\right)$, которая в ряде случаев позволяет просуммировать спектральные разложения по низкочастотным состояниям и сильно упростить выражения для амплитуд рассеяния [9].

Из процессов ИК поглощения можно выделить разрешенные, затрагивающие только колебательно-вращательные состояния примесной молекулы, и индуцированные, при которых часть энергии фотона напрямую расходуется на изменение трансляционного движения этой молекулы относительно ее окружения. В первом случае резонанс в бинарной поляризуемости не содержит трансляционной частоты, а во втором содержит и 


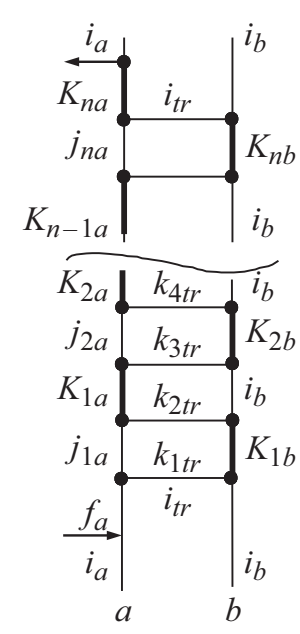

$\mathbf{A}$

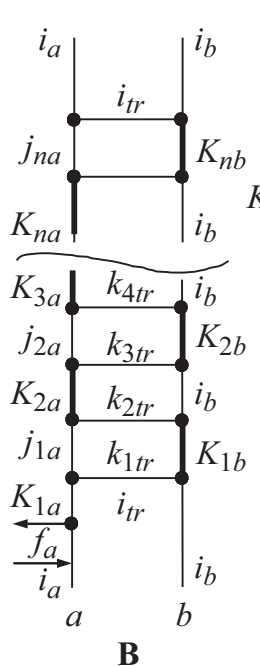

B

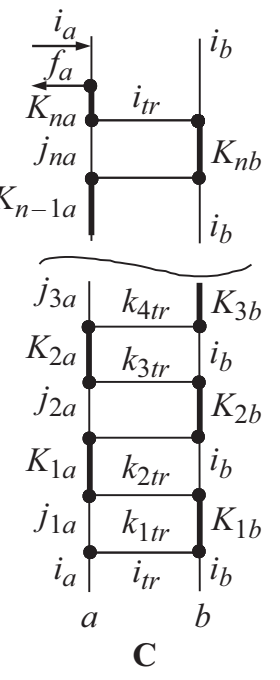

Диаграммы порядка $2 n$ по диполь-дипольному взаимодействию, соответствующие DID-компоненте резонансной части индуцированной поляризуемости. Жирные и тонкие вертикальные отрезки отвечают свободной эволюции возбужденных (прописные буквы) и основных (строчные буквы) электронных состояний молекул. Трансляционые состояния помечены индексом 'tr'. Более подробные комментарии даны в тексте статьи.

сопровожается значительным уширением индуцированных ИК полос.

Наконец, резонансы могут быть как единичными, так и многократными. Последние в данной работе не рассматриваются по причинам, упомянутым выше.

\section{Разрешенное поглощение}

Для резонансов этого типа взаимодействия молекул с фотонами должны либо предшествовать межмолекулярным DD-взаимодействиям, либо включаться после завершения последних - в противном случае резонанс будет уширен трансляционными переходами и будет относится к индуцированному поглощению. Кроме того, будем считать, что изначально заселено лишь основное состояние примесной молекулы. Принимая эти обстоятельства во внимание, получим три типа диаграмм порядка $2 n$ по DD-взаимодействиям (рисунок).

\section{Слагаемые DID (диполь-индуцированный диполь)}

Разрешенным переходам в схеме DID отвечают диаграммы, в которых колебательно-вращательные (VR) и электронные виртуальные переходы в разных молекулах чередуются как изображено на рисунке, где VR-состояния основного электронного терма обозначены строчными буквами, а возбужденные электронные состояния - прописными. Пересечение DD-сегмента приводит также и к изменению трансляционной (T) компоненты вектора состояния.
Перенос поляризации в схеме DID можно пояснить на последовательности взаимодействий, даваемых диаграммой $\mathbf{A}$ (рисунок). Вначале поглощается фотон и возбуждается дипольный момент $\mathbf{d}_{f i}$, осциллирующий на частоте $\omega_{f i}$. Далее молекула $a$ переходит в состояние $j$ и поле переходного диполя $\mathbf{d}_{j f}$ поляризует атом $b$, который совершает переходы $i_{b} \rightarrow K_{1 b} \rightarrow i_{b}$. В свою очередь электрическое поле поляризации, наведенной на атоме $b$, возмущает молекулу $a$, которая после виртуальных переходов $j_{1 a} \rightarrow K_{1 a} \rightarrow j_{2 a}$ приобретает дополнительный дипольный момент, пропорциональный ее электронной поляризуемости. Далее цикл повторяется $2 n$ раз, причем расчет показывает, что каждая пара DD-сегментов в адиабатическом пределе дает множитель $\alpha_{a} \alpha_{b} R^{-6} / 2$, где $\alpha_{k}$ суть изотропные статические электронные поляризуемости партнеров $(k=a, b)$ в их основных электронных состояниях. Для упрощения записи будем далее опускать индекс $a$.

Суммирование по низкоэнергетическим VRT-cocтояниям тривиально и диаграмме отвечает лоренцев контур с интегральной интенсивностью:

$$
\Delta A_{f i}^{(2 n)}=\left(\mathbf{d}_{i f},\left\langle f\left|\mathbf{d} \alpha^{n}\right| i\right\rangle\right)\left(\alpha_{b} R^{-6} / 2\right)^{n} .
$$

Величины $\mathbf{d}$ и $\alpha$ параметрически зависят от длины примесной молекулы. Так как поляризуемости партнеров по предположению изотропны, результат (2) имеет смысл квадрата амплитуды ИК резонанса в изотропной части индуцированной поляризуемости комплекса $a b$, а полученная поправка к интенсивности соответствует второму слагаемому выражения (1). Перестановка виртуальных состояний $K_{n a} \leftrightarrows f_{n a}$ дает дополнительную интенсивность, равную правой части (2) с переставленными индексами $i$ и $f$. С учетом того, что эрмитовы операторы d и $\alpha$ коммутируют, вклад (2) удваивается.

Наконец, диаграммы В и С (рисунок), для которых поглощение фотона непосредственно предшествует его излучению, дают такие же вклады, как и диаграмма $\mathbf{A}$, так что окончательный вклад DID порядка $2 n$ равен учетверенной правой части выражения (2). Суммирование DID-вкладов по $n$ приводит к геометрическому ряду с суммой

$$
\Delta A_{f i}^{(\mathrm{DID})}=2 R^{-6} \alpha_{b}\left(\mathbf{d}_{i f},\left\langle f\left|\mathbf{d} \frac{\alpha}{1-\alpha \alpha_{b} R^{-6} / 2}\right| i\right\rangle\right) .
$$

Поправка $\overline{\Delta A_{f i}^{(\mathrm{DID})}}$ к наблюдаемой интенсивности равна выражению (3), усредненному по трансляционной конфигурации при помощи бинарной функции распределения $g$. Последнюю для оценочных расчетов можно считать сферически симметричной: $g=g(R)$. Мы получим

$$
\begin{aligned}
& \overline{\Delta A_{f i}^{(D I D)}}=8 \pi n_{b} \alpha_{b} \int g(R) R^{-6} \\
& \quad \times\left(\mathbf{d}_{i f},\left\langle f\left|\mathbf{d} \frac{\alpha}{1-\alpha \alpha_{b} R^{-6} / 2}\right| i\right\rangle\right) R^{2} d R,
\end{aligned}
$$

где $n_{b}$ - число атомов $b$ в единице объема. Числитель в правой части (3) совпадает с предсказанием модели 
DID второго порядка, а знаменатель $1 /(1-\eta)$, где $\eta=\alpha \alpha_{b} R^{-6} / 2$, есть поправка на более высокие порядки этой модели. Подинтегральное выражение в (4) имеет резкий максимум вблизи радиуса Леннарда-Джонса $\sigma$ (так что $\eta \approx \alpha \alpha_{b} \sigma^{-6} / 2 \equiv \eta_{0}$ ), который для плотной среды близок к радиусу первой координационной сферы и должен коррелировать с радиусом $r$ ячейки ОнзагераБеттчера. Поправка интенсивности [17] на RF в ячеечной модели Онзагера-Беттчера имеет вид $\left(1-C \alpha / r^{3}\right)^{-2}$, где $C$ есть функция показателя преломления, не совпадает с (4) ни по форме, ни по содержанию, поскольку основана на некорректной процедуре усреднения (см. выше). Для случая двух атомов Фроммхольдом [7] был получен множитель $1 /(1-K(R) \eta)$, масштабирующий электронную DID-поляризуемость второго порядка с аналогичным коэффициентом $\eta$, но несколько больший $(K(R)>1)$, чем в нашем случае. Кроме того, асимптотическое разложение нерезонансной поляризуемости [7] содержит слагаемое, пропорциональное $R^{-9}$ и отсутствующее в нашем случае. Для более детального сопоставления выводов нашей теории и классической модели [7] требуется обобщить квантовые расчеты на случай нерезонансной электронной поляризуемости. В последнем случае число диаграммных вкладов значительно возрастает, что должно усиливать эффект реактивного поля по сравнению с резонансным случаем, качественно соответствуя результатам Фроммхольда [7]. Более значимым является тот факт, что оба микроскопических подхода квантовый, представленный здесь, и классический [7] дают после должного усреднения выражения, противоречащие структуре формул ячеечной модели RF.

Возможна также ситуация, когда оба фотона взаимодействуют с атомом $b$. В этом случае резонанс на частоте $i \leftarrow f$ может возникать лишь после пересечения одного или нескольких DD-сегментов, что сопряжено с возбуждением трансляционного движения. Следовательно, такие собственные диаграммы генерируют вклады, отвечающие индуцированному поглощению, представленному четвертым слагаемым в (1).

Кратко рассмотрим разрешенные вклады от несобственных диаграмм с нечетным числом $(2 n+1)$ DDсегментов, считая, как и прежде, что поляризуемость молекулы $a$ изотропна. Поскольку каждая пара сегментов не зависит от ориентации $\Omega$ вектора $\mathbf{R}$ в лабораторной системе отсчета, а каждый из сегментов содержит сферические функции второго ранга $Y_{m}^{(2)}(\Omega)$, то свертки нечетного числа таких функций дают анизотропную индуцированную поляризуемость $\Delta \alpha$, пропорциональную $Y_{m}^{(2)}$. Эта компонента поляризуемости после статистического усреднения обращается в нуль и не может вносить вклад в разрешенное поглощение света изотропной средой.

\section{Модель DID и интегральная интенсивность}

Оценим относительный вклад $(\kappa)$ эффекта DID в изменение $A_{i f}$ для основных колебательных полос мо-

\section{Таблица}

\begin{tabular}{l|c|c|c|c|c|c|c|c}
\hline \multicolumn{1}{c|}{ System } & $T, \mathrm{~K}$ & $\rho_{b}$ & $\sigma_{a b}$ & $\alpha_{b}$ & $\beta$ & $\eta_{0} \times 10^{3}$ & $\kappa$ & $\kappa_{\exp }$ \\
\hline $\mathrm{HCl} / \mathrm{LAr}$ & 90 & 770 & 6.33 & 11.1 & 6.52 & 3.02 & 0.060 & $0.39(3)$ \\
$\mathrm{HCl} / \mathrm{LKr}$ & 126 & 638 & 6.85 & 16.8 & 6.84 & 2.85 & 0.060 & $0.75(8)$ \\
$\mathrm{HCl} / \mathrm{LXe}$ & 165 & 504 & 7.27 & 27.1 & 6.46 & 3.21 & 0.063 & $1.7(3)$ \\
$\mathrm{HCl} / \mathrm{LCCl}{ }_{4}$ & 293 & 231 & 8.68 & 75.6 & 5.04 & 3.09 & 0.048 & $1.43(12)$ \\
$\mathrm{HBr} / \mathrm{LAr}$ & 90 & 770 & 6.81 & 11.1 & 8.11 & 2.66 & 0.082 & $1.0(1)$ \\
$\mathrm{HBr} / \mathrm{LXe}$ & 165 & 504 & 7.41 & 27.2 & 6.84 & 3.93 & 0.102 & $2.1(3)$ \\
$\mathrm{HBr} / \mathrm{LCCl}_{4}$ & 293 & 231 & 9.14 & 75.6 & 5.88 & 3.10 & 0.069 & 3.4
\end{tabular}

Примечание. Рассчитанные по схеме DID поправки $(\kappa)$ на изменение интенсивностей основных полос поглощения молекул $\mathrm{HCl}$ и $\mathrm{HBr}$ при помещении их в инертный растворитель, экспериментальные значения $\kappa_{\exp }[2]$ приводятся после учета эффекта внутреннего поля. Значения диаметров Леннарда-Джонса $\sigma_{a b}$ и статических поляризуемостей растворителей $\alpha_{b}$ [18] даны в а.u., $\rho_{b}$ - приведенная плотность растворителя в ед. Amagat [23].

лекул $\mathrm{HCl}$ и $\mathrm{HBr}$ при переходе в инертный раствор и сравним $\kappa$ с экспериментальным значением $\kappa_{\exp } \equiv A_{i f}^{\text {(liq) }} / A_{i f}^{\text {(gas) }}-1$. Значения электрооптических параметров (в ат. ед.), использованные в расчете, следующие: (HCl) $\alpha=17.5[18], \alpha_{f i}=0.59[19], d=0.436[18]$, $d_{f i}=0.0276[19] ;(\mathrm{HBr}) \alpha=23.9[18], \alpha_{f i}=0.76[20]$, $d=0.323$ [21], $d_{f i}=0.0115$ [21]. Гармоническое приближение $\langle f|d \alpha| i\rangle / \alpha \approx d_{f i}\left[1+d \alpha_{f i} / \alpha d_{f i}\right] \equiv d_{f i} C$ дает возможность выразить поправку к интенсивности $\kappa$ через безразмерный коэффициент $C$, равный $1.53(\mathrm{HCl})$ и $1.89(\mathrm{HBr})$.

Параметры растворителей приведены в таблице, в которой диаметры Леннарда-Джонса $\sigma_{a b}$ были вычислены по комбинационным правилам. Расчет параметра $\eta_{0}$ дает крайне малое значение (0.0030), показывающее, что поправки на реактивное поле практически исчерпываются вторым порядком по взаимодействию. Используя модель твердых сфер, можно выразить (4) через относительную DID-поправку $\kappa$ к интенсивности:

$$
\left.\overline{\Delta A_{f i}^{(D I D)}} \approx 2 \beta \eta_{0} d_{i f}\langle f|d \alpha| i\rangle\right) / \alpha=\kappa d_{i f} d_{f i},
$$

где $\beta=4 \pi n_{b} \sigma_{a b}^{3} g_{a b}\left(\sigma_{a b}\right) / 3$ есть число буферных частиц в сфере радиуса $\sigma_{a b}$, умноженное на пиковое значение бинарной функции распределения $g_{a b}\left(\sigma_{a b}\right)$. Для оценочных расчетов можно использовать типичное значение для жидкостей $g_{a b}(\sigma) \approx 2$, значения остальных параметров приведены в таблице. Отметим, что экспериментальные значения $\kappa_{\exp }$ даны с учетом поправки на эффект внутреннего поля.

Сравнение расчетов с опытом (таблица) показывает, что обратная DID-поляризация сильно недооценивает

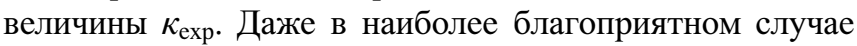
HCL-LAr $(T=90 \mathrm{~K})$ эффект RF составляет лишь шестую часть наблюдаемого усиления, а с увеличением размера частиц растворителя расхождения достигают полутора десятичных порядка. Подобное расхождение теории с опытом имеет место и для интенсивности основного тона $\mathrm{HBr}$ в инертных растворителях. 
Схожие численные результаты получаются, если применить формулы работы [2] с реалистическими значениями онзагеровского радиуса, рассчитанного по методу Линдера и Херншемайера [22]. Используя графики работ [2,17], можно заключить, что поправка интенсивности на эффект RF в таком случае становится весьма малой. Отметим, что, несмотря на некорректную с нашей точки зрения процедуру усреднения в $[2,17]$, основой расчетов, как и в нашем случае, является модель DID. Отсюда напрашивается вывод о том, что изменение интенсивности поглощения инертным окружением не связано с нерезонансным действием обратной поляризации по схеме DID.

Подобно механизму DID дисперсионные взаимодействия также могут влиять на амплитуды ИК резонансов. Дисперсионные вклады возникают при другом порядке чередования основного и возбужденных электронных состояний примесной молекулы на диаграммах (рисунок). Так, для диаграммы А нужно считать состояния $j_{n a}$ электронно-возбужденными, а состояния $K_{n a}$ отнести к колебательным подуровням основного электронного состояния. Другими словами, оба партнера одновременно совершают виртуальные переходы с активацией и дезактивацией электронной энергии. Если все подуровни основного электронного терма примесной молекулы отличаются от уровня $f$, то соответствующий дисперсионный вклад отвечает однократному резонансу на частоте $\omega_{f i}$. В противном случае возникают вклады от многократных резонансов на той же частоте, и которые в нашей терминологии относятся к индуцированным спектрам. Рассмотрение этих вкладов, как отмечалось выше, требует применения квантовой теории Лиувилля. Дисперсионные эффекты, равно как и влияние молекулярного вращения, будут проанализированы в отдельной публикации.

\section{Выводы}

Диаграммная техника является эффективным инструментом исследования механизмов, приводящих к изменению дипольного момента молекулы под влиянием ее взаимодействия с инертной средой (эффект реактивного поля). Проведенный при помощи этой техники анализ колебательных резонансов в мнимой части изотропной поляризуемости, наводимой электронными дипольдипольными взаимодействиями между молекулой и буферными частицами, позволяет классифицировать резонансные слагаемые на разрешенные (непосредственно связанные с эффектом реактивного поля) и индуцированные (сопровождающиеся изменением трансляционного движения). Показано, что точные выражения для эффекта реактивного поля, полученные по схеме дипольиндуцированный диполь (DID), противоречат полуэмпирической модели Онзагера-Беттчера. Численные оценки, однако, показывают, что DID-механизм, равно как и модель Онзагера-Беттчера с реалистическими значениями радиуса ячейки, значительно (более, чем на десятичный порядок) недооценивают величины изменения интенсивностей модельных систем (основные колебательные полосы $\mathrm{HCl}$ и $\mathrm{HBr}$ в инертных растворителях). Таким образом, для интерпретации наблюдаемых эффектов требуется разработка альтернативных моделей.

\section{Финансирование работы}

Авторы благодарят Российский фонд фундаментальных исследований за финансовую поддержку данной работы в рамках проектов 15-03-04997а и 19-03-00830а.

\section{Конфикт интересов}

Авторы заявляют, что у них нет конфликта интересов.

\section{Список литературы}

[1] Molecular Cryospectroscopy. Advances in Spectroscopy / Ed. by Clark R.J.Y., Hester R.E.. V. 23. Chap. 3. Chichester: Wiley and Sons, 1995.

[2] Буланин М.О., Бухмарина В.Н., Моисеенко Е.Г., Тохадзе К.Г. // Опт. и спектр. 1984. Т. 56. В. 5. C. 813; Bulanin M.O., Bukhmarina V.N., Moiseenko E.G., Tokhadze K.G. // Opt. Spectrosc. 1984. V. 56. N 5. P. 497.

[3] Onsager L. // J. Am. Chem. Soc. 1936. V. 58. N 8. P. 1486. doi $10.1021 / \mathrm{ja} 01299 \mathrm{a} 050$

[4] Böttcher C.J.F. Theory of Electric Polarization. Amsterdam: Elsevier, 1973. 377 p.

[5] Ландау Л.Д., Лифиии, Е.М. Теоретическая физика. Т. 5, Статистическая физика Ч. 1. М.: Наука, 1976. 583 с.; Landau L.D., Lifshitz E.M. Course of Theoretical Physics. V. 5. Statistical Physics. Part 1. Oxford: Pergamon, 1980. $562 \mathrm{p}$.

[6] Silberstein L. // Phil. Mag. 1917. V. 33. N 193. P. 92. doi $10.1080 / 14786440108635618$

[7] Frommhold L. // Adv. Chem. Phys. 1981. V. 46. P. 1.

[8] Rachet F., Chrysos M., Kouzov A. // J. Raman Spectrosc. 2003. V. 34. N 12. P. 965. doi $10.1002 /$ jrs. 1079

[9] Kouzov A.P., Chrysos M., Rachet F., Egorova N.I. // Phys. Rev. A. 2006. V. 74, N 1. P. 012723. doi 10.1103/PhysRevA.74.012723

[10] Chrysos M., Rachet F., Egorova N.I., Kouzov A.P. // Phys. Rev. A. 2007. V. 75. N 1. P. 012707. doi 10.1103/PhysRevA.75.012707.

[11] Kouzov A.P., Chrysos M. // Phys. Rev. A 2009. V. 80. N 4. P. 042703. doi 10.1103/PhysRevA.80.042703

[12] Форстер Д. Гидродинамические флуктуации, нарушенные симметрия и корреляционные функции. М: Атомиздат, 1980. 288 c.; Forster D. Hydrodynamic Fluctuations, Broken Symmetry and Correlation Functions. Redwood City: Addison-Wesley, 1990. 326 p.

[13] Mukamel S. Principles of Nonlinear Optical Spectroscopy. Oxford: Oxford University Press, 1995. 549 p.

[14] Kouzov A.P., Radi P.P. // Phys. Rev. A. 2000. V. 63. N 1. P. 010701. doi 10.1103/PhysRevA.63.010701

[15] Kouzov A., Radi P. // J. Chem. Phys. 2014. V. 140. N 19. P. 194302. doi $10.1063 / 1.4874159$ 
[16] Борн М., Воль $\oint$ Э. Основы оптики. М.: Наука, 1973. 721 с.; Born M., Wolf E. Principles of optics. Cambridge: Cambridge University Press, 1997. 987 p.

[17] Буланин М.О., Тохадзе К.Г. // Опт. и спектр. 1986. Т. 60. B. 2. C. 281; ibid. 1986. T. 61. B. 2. P. 308.

[18] Gray C.G., Gubbins K.E. Theory of Molecular Fluids. Oxford: Oxford University Press, 1984. 637 p.

[19] Maroulis G. // J. Chem. Phys. 1998. V. 108. N 13. P. 5432. doi $10.1063 / 1.475932$

[20] Cherlow J.M., Hyatt H.A., Porto S.P.S. // J. Chem. Phys. 1975. V. 63. N 9. P. 3996. doi $10.1063 / 1.431838$

[21] Kiriyama F., Rao B.S. // J. Quant. Spectrosc. Radiat. Transf. 2001. V. 69. N 5. P. 567. doi 10.1016/S0022-4073(00)00101-1

[22] Linder B., Hoernschemeyer D. // J. Chem. Phys. 1967. V. 46. N 2. P.784. doi 10.1063/1.1840740

[23] Варгафтик Н.Б. Справочник по теплофизическим свойствам газов и жидкостей. М: Наука, 1972. 720 с.; Vargaftik N.B. Tables on the Thermophysical Properties of Liquids and Gases. N.Y.: Halsted Press, 1975. 758 p. 